\title{
Seasonal forecasts for reservoir systems operation with an over-year carryover capacity - what is their value?
}

\author{
$\underline{\text { C. Arena }}^{\mathrm{a}}$, M. Cannarozzo ${ }^{\mathrm{a}}$ and M.R. Mazzola ${ }^{\mathrm{a}}$ \\ A Dipartimento di Ingegneria Civile, Ambientale, Aerospaziale e dei Materiali (DICAM), Palermo \\ University, Palermo, Italy \\ Email: claudio.arena@unipa.it
}

\begin{abstract}
Recent advances in weather and climate forecasts have brought fresh energies to studies on operation of reservoir systems with the prospect of improving their performance by assisting decisions through forecasts of water availability. Most of the studies available in the literature focus on stylized schemes with one, single- purpose, reservoir with constant demand and analyse parametrically the effect of forecasts using more or less simplified operation models. While these studies play a fundamental role in creating a general framework for analysis, there is the feeling that the diversity of the boundary conditions (climatic contexts and purposes) to the problem of reservoir management calls for more detailed analyses on specific types of reservoir systems. In this paper, we concentrate on systems with over-year behaviour that are widespread in arid and semiarid areas of the world. The question is if and in what sense streamflow forecasts can help improve reservoir operation in this type of systems, the ones that probably would most benefit from them, given their exposure to droughts.
\end{abstract}

To answer this question, we set up a real time management model of a multi-reservoir, multi-purpose system based on the rolling horizon technique (RHT). The model uses current reservoir levels and inflow forecasts over a forecasting horizon of 24 months to schedule allocations to the system's demand centres with the objective of minimizing the sum of standardized squared monthly municipal deficits and of standardized squared yearly irrigation deficits. Of this schedule, only decisions at the first time step are implemented because actual inflows become available, so that the new actual reservoir levels are available and the decision-making process can continue for the next month based on updated forecasts. We simulate the RHT over a period of 480 months in a system of two reservoirs with side irrigation demands and municipal demands in parallel. We use three types of forecasts: the two extremes of forecasting quality (average inflows and real inflows) and a simple data-driven univariate technique, denoted as Quantile Generation (QG). QG generates future inflows to the end of the current water year by equalling the quantile of cumulated inflows from the current month to the end of the water year to the quantile of the observed cumulated inflows from the beginning of the water year to the current month. We simulate the system with different demand levels, thus obtaining scenarios with different drift indexes covering a wide range of over-year storage behaviours.

We show that, perhaps not surprisingly, the RHT using forecasts of the best possible quality (real inflows) provides very similar performances, from the standpoint of total standardized squared deficits, to those obtained using worst quality forecasts (average inflows) and that the RHT with QG performs even worse than with average inflows. This would confirm that, especially in systems where the value of water is levelled out across the various demand centres, as is the case when allocation criterion is the minimization of squared standardized departures from targets, the largest value of forecasts does not reside in their ability to improve system's performances. We then explore another dimension of forecasts, by concentrating on the different ability of the RHT to provide suitable predictions of annual deficits with one-year lead when associated to the three forecast types. As expected, we find that RHT in association with real inflows is able to provide the best forecasts of actual deficits and that there is a value of the drift index above which the QG outperforms average inflows in providing forecasts of annual deficits. We conclude that the real value of forecasts in the management of this type of systems relies in their ability to provide information on future water restrictions with an advance suitable to enforce mitigation measures. If no mitigation measure is possible, then there is little scope for using forecasts to manage this type of systems. On the contrary, mitigation measures based on a timely recognition of future possible use restrictions are likely to benefit from accurate inflow forecasts. From this standpoint the QG proves a simple, promising technique to be further assessed.

Keywords: Water resources systems, rolling horizon technique, inflow forecasts, timeliness of forecasts 


\section{INTRODUCTION}

Recent advances in weather and climate forecasts have brought fresh energies to studies on operation of reservoir systems with the prospect of improving their performance by assisting decisions through forecasts of water availability. There exists now a whole body of literature containing investigations on different aspects of this problem (e.g. Georgakos and Graham, 2008, Graham and Georgakos, 2010, Zhao et al., 2011, Zhao et al., 2012). Most of the studies available in the literature focus on stylized schemes with one, single- purpose, reservoir with constant demand and analyse parametrically the effect of forecasts using more or less simplified operation models. While these works have played a fundamental role in developing a general framework for analysis by integrating forecasts with management models, there is the feeling that the diversity of boundary conditions (climatic contexts and purposes) to the problem of reservoir management calls for more detailed analyses on reservoir systems with specific behaviours. In this paper, we concentrate on systems with a significant over-year carryover capacity: they are widespread in arid and semiarid areas where long tailed yearly streamflow distributions require large storage capacities for a better exploitation of surface water resources: Vogel et al. (1999) conclude that in western US nearly all reservoirs have an over-year behaviour. According to a survey of MacMahon et al. (2007), large reservoirs in South Africa and Australia have the same characteristic, and most reservoir systems in the southern Mediterranean (Spain, Southern Italy, Greece, Turkey and North Africa) bear similar features. The question is then if and in what sense seasonal streamflow forecasts, arguably the only useful in this type of systems, can help improve their operation, as they are located prevailingly in drought-prone areas.

A previous, recent study (Arena et al., 2015) has cast doubt on the actual possibility to improve performances of these systems through seasonal streamflow forecasts. Performances are meant here, as in most studies available in the literature, as squared departures from targets in a situation where no mitigation measures are possible to modify the supply-demand balance, neither on the side of supply, by using alternative sources, neither on the side of demand thereby reducing targets. We will show that, maybe not surprisingly, in systems where the value of water is levelled out across the various demand centres, as is the case where the minimization of squared standardized departures from targets is used as allocation criterion, the largest value of forecasts does not reside in their ability to improve system's performances. If no mitigation action is possible, the benefit of forecasts, at least in this type of systems, is quite limited as they only allow displacing more effectively the use of the same water in time.

The largest value of forecasts rather seems to be found in their ability to provide information on future water restrictions with an advance suitable to enforce mitigation measures. These measures comprise all actions that can modify the supply-demand balance in a "soft" way, and hence include preparing and using a costlier supply source, informing population that certain restrictions will be in place so that they can adapt to them, rescheduling crops to account for less water availability, and so on. This aspect has seldom been addressed in the literature and this paper aims at providing a first contribution to the subject. Our experiments will be carried out on a multipurpose, two-reservoir system, using different types of forecasts: we will also introduce and analyse the merits of a data-driven streamflow forecasting procedure, denoted in the following as Quantile Generation (QG), in providing forecasts that can be valuable in the sense specified above.

\section{METHODOLOGY}

In order to understand the value of forecasts, we first set up a management procedure of a simple multi-purpose multi-reservoir system that uses streamflow forecasts. As anticipated, the system consists of two reservoirs with irrigation side demands and municipal demands in parallel (Figure 1). As a management procedure we adopt the rolling-horizon technique (RHT), consisting of making decisions about releases for the different uses at the present time step, based on current reservoirs level and on inflow forecasts for a certain number of months ahead, denoted as Forecasting Horizon (FH) (e.g. Zhao et al., 2012). Forecasts are here a vector $\widetilde{\boldsymbol{I}^{\boldsymbol{r}}}=\left[\widetilde{I_{1}^{r}}\right.$, $\left.\tilde{I}_{2}^{r} \ldots \ldots \tilde{I}_{F H}^{r}\right]$ of inflows into reservoir $\mathrm{r}$ from the current month to the forecasting horizon.

Decisions are made using a mathematical programming model of the system that optimizes a certain objective function (O.F.) subject to physical and operation constraints. Although decisions are taken for a FH stretching several months ahead, only the first decision is implemented, i.e. the current one, and the process starts again with updated information on actual reservoir storage and new inflow forecasts.

As O.F. we use a variation of the popular shortage index (e.g. Taghian et al., 2014), i.e. the sum of standardized squared deficits for the various demand centres over the FH. Deficit at a certain time $t$ for demand centre $q$ is defined as $\operatorname{Def}_{t}^{\mathrm{q}}=\mathrm{TD}_{\mathrm{t}}^{\mathrm{q}}-\mathrm{x}_{\mathrm{t}}^{\mathrm{q}}$, i.e. as the difference between target demand $\mathrm{TD}_{t}^{\mathrm{q}}$ of demand centre $\mathrm{q}$ at time $\mathrm{t}$ and allocation $\mathrm{x}$ to that demand centre at that time. If applied to systems with multiple demands, the shortage index in its original formulation leads to deficits that are proportional to demand (a proof is given in Arena et 
al, 2015). To avoid this shortcoming and ensure an equitable sharing of deficit along the different demand centres, squared deficits need to be standardised by the target demand, not by its square.

The optimization model reads as follows:

$$
\operatorname{Min}\left[\sum_{t=1}^{F H} \sum_{q=1}^{N \text { mun }} \frac{\left(\widetilde{D} e f_{t}^{q}\right)^{2}}{T D_{t}^{q}}+\sum_{y=1}^{N \text { year }} \sum_{p=1}^{\text {Nirr }} \frac{\left(\widetilde{D} e f_{y}^{p}\right)^{2}}{T D_{y}^{p}}\right]
$$

subject to:

$$
\begin{aligned}
& \widetilde{S}_{t+1}^{r}=\widetilde{S}_{t}^{r}+\widetilde{I}_{t}^{r}-\sum_{q} \widetilde{x}_{t}^{q, r}-\sum_{p} \widetilde{x}_{t}^{p, r}-\widetilde{E}_{t}^{r}-\widetilde{S}_{p} i l l_{t}^{r} \quad \forall \mathrm{r}, \mathrm{t} \\
& \widetilde{S}_{t+1}^{r} \leq K s^{r} \quad \forall \mathrm{r}, \mathrm{t} \\
& \widetilde{S P i l l}_{t}^{r} \cdot\left(\widetilde{S}_{t}^{r}-K s^{r}\right)=0 \quad \forall \mathrm{r}, \mathrm{t} \\
& \sum_{d=1}^{N f l u x} \widetilde{x}_{t}^{z, d} \leq K c^{z} \\
& \forall \mathrm{z}, \mathrm{t} \\
& \sum_{d=1}^{N f l u x(w)} \widetilde{x}_{t}^{w, d} \leq K p^{w} \\
& \forall \mathrm{z}, \mathrm{t} \\
& \sum_{r=1}^{\text {Nreservois }} \tilde{x}_{t}^{q, r} \leq T D_{t}^{q} \\
& \forall \text { q, t } \\
& \widetilde{D} e f_{t}^{p}=\widetilde{D} e f_{y}^{p} \cdot \frac{T D_{t}^{p}}{T D_{y}^{p}} \\
& \forall \mathrm{q}, \mathrm{t} \\
& \widetilde{S}_{F H}^{r} \geq S_{T}^{r} \\
& \forall \mathrm{r} \\
& \widetilde{x}_{t}^{q, r}, \widetilde{x}_{t}^{p, r}, \widetilde{S}_{t}^{r}, \widetilde{S p i l l}_{t}^{r} \geq 0 \\
& \forall \mathrm{r}, \mathrm{q}, \mathrm{p}, \mathrm{t}
\end{aligned}
$$

In (1a), $E v_{t}^{y}$ is the evaporation (in $\mathrm{Mm}^{3} /$ month) from the reservoir that is calculated as:

$$
\widetilde{E} v_{t}^{r}=a_{t}^{r}+b_{t}^{r} \cdot \widetilde{S}_{t}^{r} \quad \forall \mathrm{r}, \mathrm{t}
$$

The objective function minimizes the sum of squared deviations from monthly municipal targets in the $\mathrm{N}_{\text {mun }}$ municipal demand centres over the $\mathrm{FH}$ and the squared deviation from yearly irrigation targets in the $\mathrm{N}_{\text {irr }}$ irrigation demand centres over the $\mathrm{N}_{\text {years }}$ in the FH. This specification of the O.F. stems from the circumstance that it makes little sense considering irrigation deficits individually, on a monthly basis; rather, irrigation should be seen as a process developing consistently for several months in the water year with a peak in the driest months. In other words, situations should be avoided where the model supplies water to irrigation in a given month and completely cuts supply, say, in the next one or in the driest months of the year. To avoid or at least reduce the occurrence of these situations, in (1) yearly irrigation totals are considered and eq. (1g) prescribes that standardized irrigation deficits be the same along all periods of the water year.

As far as other constraints are concerned, equation (1a) expresses the mass balance at each reservoir between the current time step and the next one. Equation (1b) is the constraint on reservoirs' active capacity and (1c) controls that spills only take place when storage equals reservoir's capacity. Equations (1d) and (1e) constraint flows not to exceed capacity of the $\mathrm{z}$-th pipeline and of the w-th water treatment plant of the system. According to (1f) allocations should never exceed targets: (1f) is written here with reference to municipal allocation but it also applies to irrigation demand. Finally, equation (1h) constraints the end-of-period storage to be no less than a reference value that depends on the month-type of the final period (i.e. October has a different reference value than January, etc.). This reference value has been obtained by a model minimizing the same O.F. as the model presented and subject to the same constraints, but in that model the planner has a perfect foresight along the whole simulation period (40 years in this application). It should be observed that all variables bear the 
superscript $\sim$, to indicate that these are the values provided by the optimization model. As specified above, only releases corresponding to $\mathrm{t}=1$ are implemented, and the new starting storage value is obtained once actual inflow values $I_{n}$ have taken place so that the decision-making process continues for the next month. Situations can occur where actual release values differ from the planned ones due to insufficient water availability; this usually occurs when forecasts have not been able to predict correctly future inflows and storage levels are low. In these cases, real allocations differ from the planned ones: all the current water availability is shared among the different demand centres according to the allocations scheduled by model $(1)-(1 \mathrm{j})$.

\subsection{Measures of system performances}

As a measure of system performance we use the average annual standardized squared deficit. This can be defined for each municipal demand centre $\mathrm{q}$ or irrigation centre $\mathrm{p}$ as: $\operatorname{ASSD}_{\mathrm{q}(\mathrm{p})}=\frac{1}{40} \sum_{n=1}^{480}\left(\frac{D e f_{n}^{q(p)}}{T D_{n}^{q(p)}}\right)^{2}$ where 40 is the number of years of simulation. Aggregation by water use sector is obtained by summing the various ASSDs over the demand centers of interest. This performance measure differs from the objective function (1), not only because the denominator is squared, but also because irrigation deficits are evaluated individually, month by month (after, however, obtaining actual releases by considering the whole yearly irrigation demand as explained above). An alternative definition of this metrics would entail aggregating both irrigation and municipal demands (and deficits) on a yearly basis and evaluate system's performance as squared departures from yearly values.

\subsection{Application}

The model has been applied to the system of figure 1 . The RHT is simulated over a period of 480 months, from January 1971 to December 2010. Three different types of inflow forecasts are used: $\widetilde{I}_{W O R S T}^{r}$ (also termed WF hereafter) are monthly average inflows (a different value for each month-type) to reservoir $\mathrm{r}$; they are simple to obtain, but are expected to provide the lower bound of system's performances; $\widetilde{I}_{B E S T}^{r}$ (hereafter also termed $\mathrm{BF})$ are actual inflows into reservoirs along the forecasting horizon.

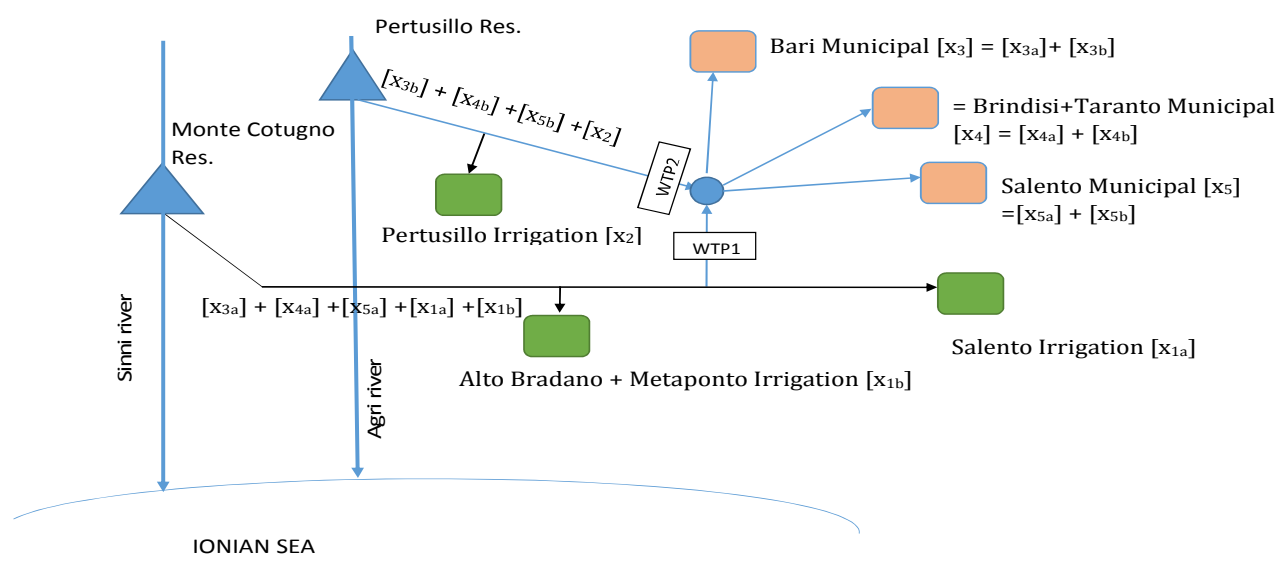

Figure 1. Schematic of the system considered. System's data: Monte Cotugno reservoir active capacity: 430 $\mathrm{Mm}^{3}$, Pertusillo reservoir active capacity: $170 \mathrm{Mm}^{3}$; Yearly irrigation targets: $\mathrm{x}_{1 \mathrm{a}}=4.5 \mathrm{Mm}^{3}, \mathrm{x}_{1 \mathrm{~b}}=160.0$ $\mathrm{Mm}^{3}, \mathrm{x}_{2}=50.0 \mathrm{Mm}^{3}$; monthly municipal targets: $\mathrm{x}_{3}=3.1 \mathrm{Mm}^{3}, \mathrm{x}_{4}=\mathrm{x}_{5}=7.3 \mathrm{Mm}^{3}$. Average inflow into Monte Cotugno reservoir: $289.4 \mathrm{Mm}^{3} /$ year, Coefficient of Variation (CV) of yearly inflows of Monte Cotugno reservoir $=0.32$, Average inflow into Pertusillo reservoir $=216.3 \mathrm{Mm}^{3} /$ year, $\mathrm{CV}($ Pertusillo $)=0.26$.

This forecast accuracy is unlikely to be achievable in practice, but, if achievable, it is expected to provide an upper bound for system's performances. In addition to these two extremes, in this paper we also explore the merits of a simple univariate, data-driven forecasting procedure, introduced by Mazzola (1994) that we shall denote as Quantile Generation (QG) in the following. This procedure predicts monthly inflows to reservoir from the current month to the end of the water year (end of September), based on the observed quantile of the cumulative inflows from the beginning of the water year (October) to the current month. 
Forecasting skills, in terms of Nash-Sutcliffe efficiency, are the following: $R_{\text {best }}^{2}(M C)=R_{\text {best }}^{2}(P E)=$ $1 ; R_{\text {worst }}^{2}(M C)=0.47, R_{\text {worst }}^{2}(P E)=0.52 ; ; R_{Q G}^{2}(M C)=0.41, R_{Q G}^{2}(P E)=0.50$ where MC denotes Monte Cotugno reservoir and PE denotes Pertusillo reservoir.

In this application, we set $\mathrm{FH}=24$ months, as this $\mathrm{FH}$ proved to be the most effective one for this specific system from the standpoint of standardized squared deficits minimization (Arena et al., 2015). In designing the vector of forecasts, it was considered that even the most recent advances in climate forecasting can produce reliable forecasts with a lead of a couple of seasons at best. For this reason, given a certain starting month, only the months to the end of the current water year are filled with forecasts pertaining to the given forecast type, while all the months left to the end of the FH are filled in with average values. For instance, if the n-th time step of simulation is a March, only values until next September are filled with either real inflows or inflows obtained from the QG method, and all months from October to the month of February of the next year are filled in with average monthly values.

In order to test the procedure over a wide range of situations, we consider four demand scenarios, obtained by a base case by increasing and decreasing municipal demand. Each scenario corresponds to a value of the drift index. The drift index m (e.g. McMahon et al., 2007) is a popular way to characterize within/over-year water resources systems behaviour. It is defined as $\mathrm{m}=(\mu-\mathrm{Y}) / \sigma$, where $\mu$ is mean yearly inflow into the system, $\mathrm{Y}$ is the yearly yield and $\sigma$ is the standard deviation of annual inflows. Systems with $m<1$ can be classified as over-year systems. In the base scenario, the value of $\mathrm{m}$ for the system with data of figure 1 is 0.75 . We then increase and decrease municipal demand (that is constant along the year), thus obtaining scenarios with $\mathrm{m}=$ $0.50, \mathrm{~m}=0.60$ and $\mathrm{m}=0.90$.

\subsection{Results}

The model has been solved using the DICOPT solver (based on the reduced gradient method) in the GAMS (General Algebraic Modeling System) platform.

Table 1 reports simulation results for the four demand scenarios (drift values) and the three forecast specifications considered. The comparison of both sectorial and system's ASSDs shows that the quality of forecasts has varying impacts on system's performances depending on the drift scenario and that the difference in system performances between best and worst forecast tends to increase when drift increases, implying that when pressure on resources is very high (as in the case of lower drift values) there is little scope for managing resources, regardless of the quality of forecasts. For low drift values, the QG method performs even worse than average values.

Table 1. Annual standardized squared deficit per sector and for the whole system for four values of the drift index $(\mathrm{m})$ and for three different types of inflow forecasts: average values (worst forecast: WF), Quantile Generation (QG) and real inflows (best forecast: BF)

\begin{tabular}{|c|c|c|c|c|c|c|c|c|c|c|c|c|}
\hline & \multicolumn{3}{|c|}{$\mathrm{m}=\mathbf{0 . 5 0}$} & \multicolumn{3}{|c|}{$\mathrm{m}=\mathbf{0 . 6 0}$} & \multicolumn{3}{|c|}{$\mathrm{m}=\mathbf{0 . 7 5}$} & \multicolumn{3}{|c|}{$\mathrm{m}=\mathbf{0 . 9 0}$} \\
\hline & WF & QG & BF & WF & QG & BF & WF & QG & BF & WF & QG & BF \\
\hline & & \multicolumn{11}{|c|}{ Irrigation demand $\left[\mathrm{x}_{1 \mathrm{a}}\right]+\left[\mathrm{x}_{1 \mathrm{~b}}\right]+\left[\mathrm{x}_{2}\right]$} \\
\hline Average annual supply $\left[\mathrm{Mm}^{3}\right]$ & 180.3 & 181.5 & 181.8 & 197.4 & 198.2 & 199.3 & 205.2 & 206.2 & 206.5 & 208.3 & 209.4 & 209.8 \\
\hline Average annual deficit $\left[\mathrm{Mm}^{3}\right]$ & 34.2 & 33.0 & 32.7 & 17.1 & 16.3 & 15.2 & 9.3 & 8.3 & 8.0 & 6.2 & 5.1 & 4.7 \\
\hline \multirow[t]{2}{*}{ ASSD } & 48.2 & 50.6 & 44.3 & 26.5 & 28.3 & 21.9 & 17.0 & 17.5 & 12.6 & 12.2 & 11.4 & 7.6 \\
\hline & & \multicolumn{11}{|c|}{ Municipal demand $\left[\mathrm{x}_{3}\right]+\left[\mathrm{x}_{4}\right]+\left[\mathrm{x}_{5}\right]$} \\
\hline Average annual supply $\left[\mathrm{Mm}^{3}\right]$ & 257.7 & 254.2 & 257.8 & 229.1 & 225.3 & 228.3 & 203.1 & 200.5 & 201.7 & 180.8 & 179.2 & 179.8 \\
\hline Average annual deficit $\left[\mathrm{Mm}^{3}\right]$ & 51.2 & 54.8 & 51.1 & 20.0 & 23.8 & 20.8 & 8.9 & 11.5 & 10.3 & 4.8 & 6.4 & 5.8 \\
\hline \multirow[t]{2}{*}{ ASSD } & 83.2 & 97.3 & 85.5 & 31.4 & 39.3 & 33.3 & 18.1 & 22.9 & 20.1 & 11.4 & 14.4 & 12.4 \\
\hline & & \multicolumn{11}{|c|}{ System's demand (Irrigation + Municipal) } \\
\hline Average annual supply $\left[\mathrm{Mm}^{3}\right]$ & 438.1 & 435.6 & 439.7 & 426.5 & 423.5 & 427.6 & 408.3 & 406.6 & 408.2 & 389.1 & 388.6 & 389.6 \\
\hline Average annual deficit $\left[\mathrm{Mm}^{3}\right]$ & 85.4 & 87.8 & 83.8 & 37.1 & 40.1 & 36.0 & 18.2 & 19.8 & 18.3 & 11.0 & 11.6 & 10.5 \\
\hline ASSD & 131.4 & 147.9 & 129.8 & 57.9 & 67.6 & 55.1 & 35.1 & 40.3 & 32.7 & 23.6 & 25.8 & 19.9 \\
\hline
\end{tabular}

Given these results, one could provocatively conclude that developing real - time procedures based on forecasts may not be very beneficial in over-year systems. However, the above analysis has overlooked a fundamental function of forecasts, i.e. enabling the effective implementation of mitigation measures that are able to modify the water balance either on the side of demand or on the side of supply, or on both, in the sense specified in the introduction. The point here is that, as shown in table 1 , forecasts of different quality may eventually lead to 
the same system's performances, implying that this type of performance is not able to capture the whole value of forecasts. This value is revealed instead by the ability of better quality forecasts to predict future shortages with suitable advance, as will be shown next.

Figure 2 shows forecasts of system's annual deficits provided by the three forecast specifications and compares them with the pattern of actual deficits along the 40-year simulation period.
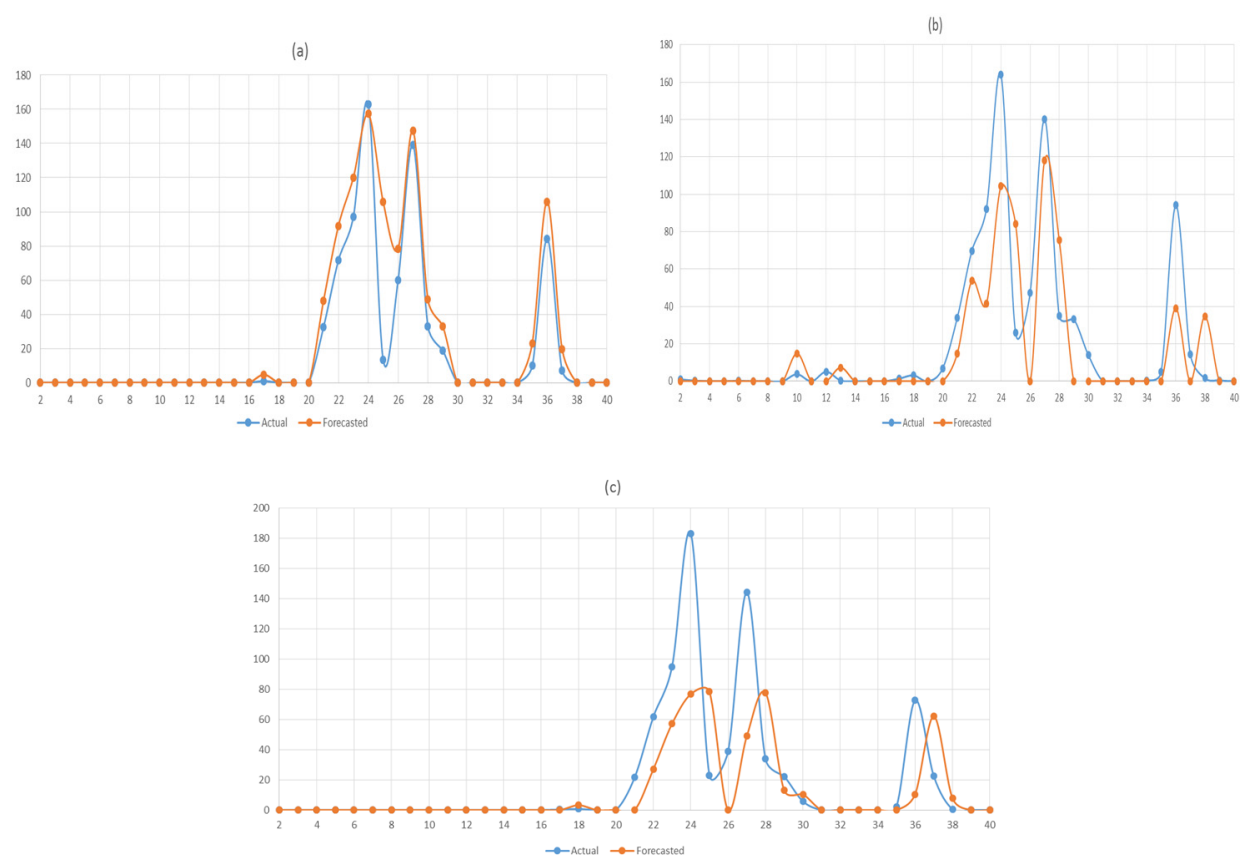

Figure 2. Pattern of actual (blue) and forecasted with one-year lead (orange) annual system's deficits (in $\mathrm{Mm}^{3}$ ) along the 40 year simulation period for $\mathrm{m}=0.75$, using (a) best forecasts, (b) quantile generation, (c) worst forecasts.

It should be remarked that, albeit similar, even the pattern of actual deficits depends on the type of forecasts used. Figure 2 shows that real inflows yield the best forecasts of deficits and average values the worst ones, while QG stays somewhere in the middle. Incidentally, it should be highlighted the figure shows how the system is affected by multi-year droughts that severely challenge water supply. In order to allow a more objective comparison of the performances provided by the different forecasts, table 2 contains the average squared deviations of one-year lead forecasted annual deficits from the actual one, for the different drift values considered.

Table 2. Average squared deviations of one-year lead forecasted system's deficits from real system's deficits.

\begin{tabular}{|c|c|c|c|c|c|c|c|c|c|c|c|}
\hline \multicolumn{3}{|c|}{$\mathrm{m}=0.50$} & \multicolumn{3}{|c|}{$\mathrm{m}=0.60$} & \multicolumn{3}{|c|}{$\mathrm{m}=0.75$} & \multicolumn{3}{|c|}{$\mathrm{m}=0.90$} \\
\hline $\mathrm{BF}$ & $\mathrm{QG}$ & $\mathrm{WF}$ & $\mathrm{BF}$ & QG & $\overline{\mathrm{WF}}$ & $\mathrm{BF}$ & $\overline{Q G}$ & WF & $\overline{B F}$ & $\overline{\mathrm{QG}}$ & WF \\
\hline 251.6 & 1760.8 & 1374.2 & 304.8 & 1157.2 & 1159.3 & 402.7 & 510.0 & 860.0 & 380.0 & 277.0 & 582.5 \\
\hline
\end{tabular}

Results of table 2 draw a more complex picture than the one portrayed in figure 1 . In the first place, while best forecasts yield always best performances compared to worst ones, QG has a less clear behaviour: for high drift indexes, it even seem to outperform best forecasts in predicting annual deficits. However, a more detailed analysis (not shown here for reasons of space) shows that squared deviations of deficits obtained with BF are heavily influenced by a single outlying value at year 25 (also visible in Figure 1 (a)) for 1b, the largest irrigation centre of the whole system. Without it, average squared deviations of forecasted deficits from real ones using $\mathrm{BF}$ would be considerably lower.

This notwithstanding, it is confirmed that QG significantly outperforms average inflows as forecasts at least for drift values $\geq 0.75$, while for drift values lower than 0.75 the performances of QG tend to decrease. In extreme conditions such as the ones portrayed by $\mathrm{m}=0.50$, QG is outperformed by average inflows, thereby confirming results of table 1 that however focused on annual standardized squared deficits. In conclusion, there is a range of systems for which the QG method, although performing poorly when it comes to allocate resources 
along the forecasting horizon, shows a better ability than average values to provide predictions on deficits with a one-year lead.

\section{CONCLUSIONS AND RECOMMENDATIONS}

The paper has provided some insights into the value of forecasts for managing reservoir systems with overyear behaviour, by simulating the operation of a two-reservoir system for municipal and irrigation supply over a 40-year period, supported by forecasts of different quality. The criterion used to manage the system is to minimize the sum of standardized squared deviation from target releases for the six demand centres. To increase the generalizability of results, simulations have been carried out for four different demand scenarios, ranging in the whole domain of over-year behaviour, from high pressure on resources (corresponding to a drift index $\mathrm{m}$ of 0.50$)$, to moderate pressure on resources $(\mathrm{m}=0.90)$. Results confirm that, albeit different, the performances of the management model do not differ considerably even when using the two extremes of forecasting ability, i.e. real inflows (best forecasts - not achievable in practice) and average inflows (worst forecasts). This suggests that, if it is impossible to adjust the supply-demand balance in the short term, forecasts can only help mitigate the effect of unavoidable shortages, but overall this has little impact because their effect is simply to displace the use of the same water into a different time step of long, multi-year drought periods. The type of performance criterion adopted can influence this result and it is likely that other criteria, assigning different values to resources according to their use, may change this picture. However, we argue that the true value of forecasts does not reside in their ability to mitigate (unavoidable) restrictions, rather in the support they can provide to plan effectively measures, on the side of both demand and supply, to modify the water balance of the system. While integration of these measures in the model is a fundamental, next step of research to compare the effectiveness of different types of forecasts, in the paper we relate this ability to support mitigation measures to the ability of the different forecast types to provide predictions on deficits with suitable advance (one year). From this standpoint, first results show that the model run with the Quantile Generation method is capable of providing closer predictions of annual deficits than average inflows for systems with drifts higher than 0.75 , while in the scenarios where pressure on resources grows (and the drift index is lower), the QG method loses its forecasting ability.

\section{REFERENCES}

Arena, C., Cannarozzo, M., and M.R. Mazzola (2015). Exploring the potential and the boundaries of the rolling horizon approach for the management of reservoir systems with over-year carryover capacity. Paper presented at European Water Resources Association (EWRA) 9th World Congress, EWRA, Istanbul, Turkey, May 10-13.

Georgakos, K.P., and N.E. Graham (2008). Potential Benefits of Seasonal Inflow Prediction Uncertainty for Reservoir Release Decisions, Journal of Applied Meteorology and Climatology, 47, 1297-1320.

Graham, N.E., and K.P. Georgakakos (2010). Toward Understanding the Value of Climate Information for Multiobjective Reservoir Management under Present and Future Climate and Demand Scenarios, Journal of Applied Meteorology and Climatology, 49, 557-573.

Mazzola, M. R. (1994). Multipurpose reservoir real time management by stochastic interannual forecasting model. Proceedings Second European Conference on "Advances in Water Resources Technology and Management”, edited by: Tsakiris, G. and Santos, M. A. Balkema, Rotterdam.

McMahon, T.A., Pegram G. G.S, Vogel, R.M., and Peel, M.C. (2007). Revisiting reservoir storage-yield relationships using a global streamflow database, Advances in Water Resources 30 (2007), 1858-1872.

Taghian, M., Rosbjerg, D., Haghighi, A., and H. Madsen (2014). Optimization of Conventional Rule Curves Coupled with Hedging Rules for Reservoir Operation, Journal of Water Resources Planning and Management ASCE, 140, 693-698.

Zhao, T. T. G., Cai, X. M., and D. W. Yang (2011). Effect of streamflow forecast uncertainty on real-time reservoir operation, Advances in Water Resources, 34, 495-504, doi:10.1016/j.advwatres.2011.01.004.

Zhao, T., Yang, D., Cai, X., Zhao, J., and H. Wang. (2012). Identifying effective forecast horizon for real-time reservoir operation under a limited inflow forecast, Water Resources Research, 48, W01540, doi:10.1029/2011WR010623.

Vogel, R.M., Lane, M., Ravindiran, R.S., and P. Kirshen (1999). Storage reservoir behavior in the United States, Journal of Water Resources Planning and Management ASCE, 125(5), 245-254. 